\title{
Resúmenes del Premio Académico «Dr. Mario Shapiro»
}

\author{
«Dr. Mario Shapiro» Academic Award Abstracts \\ Resumo do Prêmio Acadêmico «Dr. Mario Shapiro»
}

\section{Subdiagnóstico de la lesión renal aguda en pacientes obstétricas complicadas en la Unidad de Cuidados Intensivos Estrada-Gutiérrez Alfonso, Maya-Contreras César, \\ Aguilar-Arciga Themis Gwendolyne, García-Gómez Angélica, Barriga-Ferreyra Pedro \\ Hospital de la Mujer Ocolusen, Michoacán. Hospital Innova Médica, Morelia, Michoacán. Hospital General "Dr. Miguel Silva», Morelia, Michoacán, México.}

Objetivo: Definir la prevalencia y factores asociados de lesión renal aguda en el embarazo en la Unidad de Cuidados Intensivos del Hospital de la Mujer, Morelia, Michoacán, México. Diseño: Estudio retrospectivo, transversal y descriptivo de enero 2013 a agosto 2018; 213 expedientes de pacientes obstétricas complicadas. Criterios de inclusión: pacientes obstétricas complicadas que ingresaron a la $\mathrm{UCl}$, pacientes que cumplieron criterios para lesión renal aguda. Criterios de exclusión: lesión renal crónica, expediente clínico no disponible. Sólo 154 cumplieron con los criterios de selección. Resultados: Se incluyeron 154 pacientes obstétricas complicadas; un promedio de $25.6 \mathrm{p} \pm$ 1.6 por año. El 36\% tuvo diagnóstico de eclampsia; 35.3\% preeclampsia; $29.3 \%$ síndrome de HELLP; $19.3 \%$ hemorragia obstétrica, $10 \%$ sepsis. Se demostró asociación de PR-AKI con síndrome de HELLP $(p=0.0003)$ y preeclampsia $(p=0.01)$. Se encontró un subdiagnóstico del $36.7 \%$ al buscar PR-AKI utilizando los criterios RIFLE y AKI ( $p=$ 0.000007 ). De las pacientes con PR-AKI grado 3, 20\% requirió terapia de reemplazo renal continua. Conclusiones: La lesión renal asociada al embarazo complicado tiene una prevalencia de 6.7\%. Las complicaciones asociadas con PR-AKI son síndrome de HELLP y preeclampsia. La PR-AKI está subdiagnosticada hasta en $36.7 \%$.

\section{Asociación de ferritina al ingreso, con el deterioro ventilatorio y mortalidad en pacientes con enfermedad por COVID-19, ingresados en terapia intensiva \\ Galicia García César, Santana Hernández Gabriela Patricia, Vega Sánchez Ángel Emmanuel \\ Hospital San Ángel Inn Universidad, Ciudad de México.}

Introducción: Se realizó un estudio retrospectivo, transversal, en el cual se reclutaron 48 pacientes en la terapia intensiva del Hospital San Ángel Inn Universidad. Se tomaron los datos clínicos y variables de interés del expediente clínico. Se realizó una descripción de los datos con base en su distribución en medias con desviación estándar o medianas con rangos intercuartilares de las variables continuas y los datos categóricos en frecuencias con porcentaje. El objetivo del estudio fue evaluar la ferritina sérica medida al ingreso como predictor de evolución en la $\mathrm{UCl}$, para lo cual se tomaron como desenlaces principales intubación y muerte. Se realizó un cálculo de sensibilidad y especificidad, así como el área bajo la curva por medio de curva ROC. Se evaluó la fuerza de asociación de la ferritina por medio de cálculo del OR crudo y ajustado por regresión logística univariada multivariada. Se evaluó la ferritina como marcador pronóstico de intubación y mortalidad en pacientes ingresados en terapia intensiva, para mortalidad se escogió la curva ROC con un valor de sensibilidad de $50 \%$, especificidad 88.89 , LR + de 4.5 y LR - 0.56; con ello, el área fue de 8 , utilizando este valor de corte, la proporción de pacientes con mortalidad bajo este valor fue de 7.8, para riesgo de mortalidad y sensibilidad de $72.73 \%$, especificidad 73.08 , LR + de 2.7 y LR -0.37 , tras lo cual el área fue de 7.2, utilizando este valor de corte, la proporción de pacientes intubados bajo este valor fue de 9.3, con ello se

www.medigraphic.com/medicinacritica realizó una regresión logística univariada observado un OR crudo de 8 y ajustado de 7.8 para mortalidad, y OR crudo de 7.23 y ajustado de 9.3 para intubación, ajustada a confusores para edad con OR 1.0, con valor de $p$ de 0.080 , hipertensión con OR de 3.2 , valor de $p$ de 0.10 y para obesidad con OR de 1.89, con valor de $p$ de 0.041 . Conclusión: En los pacientes ingresados al Hospital San Ángel Inn Universidad con criterios de gravedad para terapia intensiva, la presencia de ferritina mayor a $1,100 \mathrm{ng} / \mathrm{mL}$ tiene una sensibilidad de $72.73 \%$, especificidad de $73.08 \%$ y LR + de 2.70 y LR - de 0.37 de este valor se asoció con intubación con OR crudo de 7.2 y ajustado de 9.3 y la presencia de ferritina mayor a $2,507 \mathrm{ng} / \mathrm{mL}$ tiene una sensibilidad de $50 \%$, especificidad de $88.89 \%$ y LR + de 4.5 , LR - de 0.56 de este valor se asoció con mortalidad con OR crudo de 8 y ajustados de 7.8. Por tal motivo, un valor de corte de ferritina superior a 1,100 en pacientes ingresados a $\mathrm{UCl}$ debe ser considerado como un pronóstico para intubación durante el internamiento. Este marcador debe ser evaluado con mayor número de pacientes para comprobar su utilidad.

Terapia prono y supervivencia en SARS-CoV-2 en cuidados intensivos de un hospital de tercer nivel de atención en México Valencia Rosas Andrés, González Pérez Netzahualcóyotl,

López Carrillo Lilia

Centro Médico ISSEMyM.

Introducción: La posición en decúbito prono mejora la supervivencia en pacientes con síndrome de dificultad respiratoria aguda (SDRA) moderado o grave, por lo que es importante determinar el impacto de esta estrategia en pacientes con SARS-CoV-2. Metodología: Estudio observacional, retrospectivo, longitudinal, comparativo, en pacientes con SARS-CoV-2, que requirieron ventilación mecánica por SDRA. Se calculó la probabilidad de supervivencia individual acumulada a lo largo de 60 días de seguimiento con el método de Kaplan-Meier, en grupos tratados con soporte multiorgánico aislado o asociado con terapia prono. Resultados: Se reclutaron 82 pacientes, $62(75.6 \%)$ requirieron terapia prono por una relación $\mathrm{PaO}_{2} / \mathrm{FiO}_{2}<150 \mathrm{mmHg}$, mientras que $20(24.4 \%)$ no fueron sometidos a esta estrategia por presentar una relación $\mathrm{PaO}_{2} / \mathrm{FiO}_{2}$ $>150 \mathrm{mmHg}$. La supervivencia a 60 días fue de 54.8 y $80 \%$, respectivamente ( $p$ 0.069). Conclusión: La terapia prono en pacientes con SARSCoV-2 con una relación $\mathrm{PaO}_{2} / \mathrm{FiO}_{2}<150 \mathrm{mmHg}$ es una estrategia que permite mantener una supervivencia equiparable a aquella que tienen los pacientes que ingresan con una $\mathrm{PaO}_{2} / \mathrm{FiO}_{2}$ superior a $150 \mathrm{mmHg}$.

Es el índice neutrófilos /linfocitos un predictor de mortalidad, en paciente con infección por SARSCoV-2 en la Unidad de Cuidados Intensivos del Hospital Regional «Lic. Adolfo López Mateos» Montalvo Aguilar Michell, González López César Augusto Médicos del Hospital Regional "Lic. Adolfo López Mateos", ISSSTE.

Introducción: En diciembre de 2019 se dio a conocer que en la ciudad de Wuhan (China) se había presentado una nueva serie de casos de neumonía, al parecer originados por un nuevo tipo de coronavirus. El 07 de enero de 2020 , el nuevo coronavirus fue anunciado oficialmente por las autoridades chinas como el agente causal de dichas infecciones. El índice neutrófilos/linfocitos (INL) ha tomado interés como biomarcador sérico para definir la gravedad. CataudeIla y colaboradores reportaron la relación proporcional del INL con la gravedad de la neumonía y algunos desenlaces adversos; además, se ha observado que puede ser predictor de mortalidad en Unidades de Cuidados Intensivos. Métodos: Se realizó un estudio prospecti- 
vo, longitudinal, analítico en el que se ingresaron todos los pacientes con diagnósticos de infección por SARS-CoV-2 en un periodo de tiempo de abril a agosto 2020, en la Unidad de Cuidados Intensivos del Hospital Regional «Lic. Adolfo López Mateos». Resultados: La población estudiada se compuso de 59 sujetos; no obstante, para el análisis se excluyeron dos pacientes por datos insuficientes. De los 57 restantes, $61.4 \%(n=35)$ fueron hombres y $38.6 \%(n=22)$ mujeres. Con respecto a la curva de ROC, la cual es un análisis cuya finalidad determina un valor de corte con alta sensibilidad a predecir un suceso (en nuestro objetivo: mortalidad), se realizó y arrojó un resultado significativo sólo en el índice neutrófilos/linfocitos cuando el paciente se encontraba en la $\mathrm{UCI}$ con un área bajo la curva de 0.728 (IC: $0.597-0.860)(p=0.004)$, mostró una especificidad de $80 \%$ cuando el índice tenía un valor de 10.35 o mayor en estos pacientes. El cálculo para este parámetro, mientras que el paciente ingresó a urgencias, no fue significativo y la hipótesis se negó con un área bajo la curva de 0.628 (IC 0.480-0.777). Conclusiones: Entre nuestros resultados, destaca el valor reportado promedio en los pacientes que fallecieron durante su estancia en la UCl, la cual fue de $54.56 \pm 10.89$ años vs aquellos vivos que fue de $48.23 \pm 11.42$ ( $p=0.037$ ), lo que concuerda con lo reportado en la literatura por diversos autores, se decidió buscar algún punto de corte en población mexicana, donde Reyes-Gálvez y su grupo reportaron en sepsis que un valor de $18.1 \mathrm{a}$ 36 , o incluso mayor, es un valor del índice neutrófilo/linfocito que se asocia con un estado severo o crítico de la enfermedad y se asocia de forma modesta con la escala de APACHE. De acuerdo con estos puntos de corte, la población que se analizó no reportó asociaciones significativas mientras que se encontraban en los Servicios de Urgencias, posiblemente porque la respuesta inflamatoria aguda aún no se desarrollaba en su totalidad, o la sintomatología primaria sólo es insuficiencia respiratoria que ameritaba su hospitalización y posterior pase a la $\mathrm{UCl}$, donde este valor fue significativo $(p=0.022)$.

\section{Relación de hipercloremia con mortalidad} en pacientes postoperados electivamente de resección de tumores intracraneales

Ramírez García Héctor Alejandro, Salas Delgado Arnoldo IMSS UMAE No. 25, Monterrey, Nuevo León, México.

Antecedentes: La hipercloremia en el paciente neurocrítico es frecuente y se ha asociado con múltiples desenlaces no favorables. Objetivo: Establecer la relación entre el nivel sérico de hipercloremia en el periodo postoperatorio inmediato y la mortalidad a corto plazo en pacientes neuroquirúrgicos posterior a resección de tumoración intracraneal de manera electiva. Métodos: Se realizó un estudio retrospectivo, observacional, comparativo, longitudinal en la Unidad de Terapia Intensiva de un hospital de tercer nivel. Se obtuvieron los datos de los pacientes adultos que se sometieron a un procedimiento electivo de neurocirugía para resección de una masa tumoral intracraneal del 01 de enero del 2016 al 31 de diciembre del 2018 y que estuvieron al menos 24 horas en la UCIA en el postoperatorio inmediato. Los pacientes fueron clasificados de acuerdo con el nivel más alto de cloro sérico en las primeras 24 horas. El punto de comparación fue la mortalidad a 30 días de admisión. Resultados: Se incluyeron 122 pacientes, mediana de edad 48.7 años (2084), 65 hombres (53\%). Hipercloremia en 61 pacientes (50\%). Los niveles promedio de cloro en la población general fueron de 110.47 $\pm 4.7 \mathrm{mmol} / \mathrm{L}$. Hubo una prevalencia en todo el grupo de $50 \%$ en el periodo postoperatorio inmediato, de los cuales, 12 fallecieron durante los siguientes 30 días. Hubo un marcado incremento de los niveles de cloro sérico en el grupo de mortalidad (60\%, comparado con el grupo de no mortalidad, 48\%) sin lograr una correlación significativa $(p=0.32)$. No hubo una correlación significativa entre la presencia de hipercloremia como una variable continua $(p=0.35)$ o categórica en el periodo postoperatorio inmediato y la mortalidad a 30 días. Conclusiones: La hipercloremia sérica en las primeras 24 horas en el grupo postoperatorio neurocrítico no se relaciona de manera significativa a 30 días con la mortalidad.
Hipoxemia y mecánica ventilatoria en pacientes con infección por coronavirus 2-síndrome respiratorio agudo grave

Ramírez Urízar Diego Andrés, Aisa Álvarez Alfredo,

Franco Granillo Juvenal, Aguirre Sánchez Janet, Luján Sitt Uriel Elías, Vigil Escalera María

Centro Médico ABC, Ciudad de México, México.

La pandemia de SARS-CoV-2 ha inspirado un nuevo interés en los fundamentos de la patología del síndrome de distrés respiratorio del adulto (SDRA) asociado con COVID-19. Este estudio busca las causas de hipoxemia y su mecánica ventilatoria en los pacientes en ventilación mecánica por COVID-19. Método: Estudio de cohorte prospectivo. Del 23 de marzo al 15 de mayo de 2020 se recolectó información basal (comorbilidades, estudios de laboratorio y escalas pronósticas) y parámetros ventilatorios y gasométricos de todos los pacientes mayores de 18 años que recibieron ventilación mecánica por COVID-19 y se dio seguimiento hasta el día 15 de ventilación mecánica (VM). Resultados: Se excluyeron 13 pacientes por expedientes incompletos. Un total de 63 pacientes con distensibilidad pulmonar de $41.44 \pm 12.18 \mathrm{~mL} / \mathrm{cmH}_{2} \mathrm{O}$, driving pressure (DP) de $12.18 \pm 2.67 \mathrm{cmH}_{2} \mathrm{O}$, volumen tidal $(\mathrm{Vt})$ de $7.02 \pm 1.11 \mathrm{~mL} / \mathrm{kg}$ de peso predicho, cortocircuitos pulmonares de $23.38 \%$ (3.23-90.05), gradiente alveoloarterial $354.96 \pm 75.37 \mathrm{mmHg}$ y relación $\mathrm{PaO}_{2} / \mathrm{FiO}_{2}$ de $129(48-309) \mathrm{mmHg}$. El análisis lineal de medidas repetidas a la evaluación de los pacientes que presentaron extubación temprana versus VM prolongada, con diferencias estadísticamente significativas en Vt y DP ( $p=0.04, p=0.0005$, respectivamente) si elevan más de $7.5 \mathrm{~mL} / \mathrm{kg}$ y $13 \mathrm{cmH}_{2} \mathrm{O}$, respectivamente. Conclusiones: $\mathrm{La}$ hipoxemia en estos pacientes se debe a dos causas: aumento de cortocircuitos pulmonares y desequilibrio de ventilación/perfusión pulmonar. Se debe mantener una DP menor que $13 \mathrm{cmH}_{2} \mathrm{O}$ y un Vt menor que $7.5 \mathrm{~mL} / \mathrm{kg}$ en todos los días de VM.

\section{Estatus de la vitamina D en sepsis en la}

Unidad Cuidados Intensivos Adultos

Héctor Antonio López Morán, ${ }^{*}$ Arturo Razcón Echeagaray,,

Luis Barrientos Quintanilla, ${ }^{\ddagger}$ Víctor Manuel Sánchez Nava,

Carlos Eduardo Chávez Pérez ${ }^{\ddagger}$

*Hospital Ángeles Valle Oriente. ¥Hospital San José, Hospital

Metropolitano. §Hospital San José y Hospital Zambrano Hellion.

Introducción: En la actualidad, se ha dado gran importancia al estudio de vitaminas $C$ en sepsis. Sin embargo, especialmente en el contexto de cuidados intensivos, existe evidencia limitada pero significativa de la elevada prevalencia de la deficiencia de vitamina $\mathrm{D}$ en los enfermos con sepsis y choque séptico, correlacionándose con la gravedad y la probabilidad de disfunción orgánica. Material y métodos: Estudio retrospectivo, monocéntrico, observacional y descriptivo, muestra a conveniencia en un hospital de tercer nivel. Se analizó un total de 492 pacientes, de los cuales, 105 cursaron con el diagnóstico de sepsis, y de éstos 30 contaron con medición de vitamina $\mathrm{D}$. La edad media fue de $56 \pm 15$ años, predominó el sexo masculino (70\%), antecedentes de patología oncológica $(26.7 \%)$ e inmunosupresión (30\%). Al ingreso a la Unidad de Cuidados Intensivos $73.3 \%$ se encontraron en estado de choque, $70 \%$ bajo ventilación mecánica, con un promedio en las escalas de mortalidad de 16 puntos en APACHE II, con $25 \%$ de mortalidad de 8 puntos en SOFA con $15-20 \%$ de mortalidad. De acuerdo con los niveles de vitamina $\mathrm{D}$, se encontraron dos grupos: insuficiencia (menor de $10 \mathrm{ng} / \mathrm{mL}$ ) y deficiencia (mayor a $10 \mathrm{ng} / \mathrm{mL}$ ). Resultados: No se encuentra diferencia significativa en edad $(p=0.724)$, estancia intrahospitalaria $(p=0.755)$, SOFA $(p=0.241)$. Existe significancia estadística en APACHE II con un puntaje medio de 19.5 puntos en el grupo menor de $10 \mathrm{ng} / \mathrm{mL}$ (mortalidad esperada de 25\%) y de 14.3 puntos en el grupo mayor a $10 \mathrm{ng} / \mathrm{mL}$ (mortalidad esperada de $15 \%$ ). La mortalidad se estima en $42.9 \%$ en el grupo menor de 10 y de 12.5 en el grupo mayor de 10 , pero no es significativo estadísticamente. Conclusiones: No existe correlación con un valor estadísticamente 
significativo entre los niveles de vitamina $D$ y días de estancia intrahospitalaria. El porcentaje de defunción de los pacientes con valor inferior a $10 \mathrm{ng} / \mathrm{mL}$ es de $42.9 \%$, a diferencia de los pacientes con valor superior a $10 \mathrm{ng} / \mathrm{mL}$ de $12.5 \%$, que no alcanza un valor estadísticamente significativo; sin embargo, la población estudiada es pequeña, por lo que únicamente se requiere aumentar el tamaño de muestra para obtener un valor estadísticamente significativo.

\section{Uso de ácido ascórbico en pacientes con traumatismo craneoencefálico severo y su impacto en el tiempo de estancia en la Unidad de Cuidados Intensivos Adultos \\ Macías Limón Juan Diego de Jesús, Hernández Plata Alma Erika, Bravo Santibáñez Edgar, Hernández Martha Alicia \\ UMAE, CMN Bajío No. 1, IMSS. León, Guanajuato, México.}

Introducción: El traumatismo craneoencefálico (TCE) es la principal causa de muerte y discapacidad en adultos jóvenes alrededor del mundo. Se ha demostrado que el manejo orientado a limitar la lesión secundaria impacta sobre la morbilidad y mortalidad de esta entidad. Esta etapa de lesión secundaria se desarrolla con la activación de múltiples vías moleculares y celulares; ocasionando edema cerebral, aumento en la presión intracraneal y, finalmente, disminución en la presión de perfusión cerebral. Existe además gran liberación de radicales libres de oxígeno, como la causa directa de este daño. Las medidas de neuroprotección están encaminadas al mantenimiento de las presiones de perfusión cerebral adecuadas, manejo de la hipertensión intracraneal y prevención de la hipotensión, hipoxia y convulsiones. Los mecanismos de defensa contra el daño celular secundario a estrés oxidativo se encuentran dañados en enfermedad aguda, debido a la depleción de los reductores de radicales libres como la vitamina $\mathrm{C}$ o ácido ascórbico. Se ha observado que en pacientes con TCE, las concentraciones plasmáticas de ácido ascórbico se encontraron bajas. En adición a su potente acción como reductor de radicales libres, el ácido ascórbico puede estabilizar el endotelio y promover la integridad de la barrera hematoencefálica (BHE). Posterior al impacto mecánico del TCE, isquemia, reperfusión y el desequilibrio entre disminución de factores protectores como reductores de radicales libres y factor 2 relacionado NF-E2 y un aumento en los factores de daño como la metaloproteinasa (MMP-9), que llevan a la disrupción de las proteínas de uniones fuertes transmembrana en la lámina basal de la BHE, lo que resultó en disminución del edema perilesional en la imagen. Objetivo: Determinar la utilidad del ácido ascórbico en pacientes con TCE severo y su impacto en el tiempo de estancia hospitalaria en la Unidad de Cuidados Intensivos Adultos (UCI). Resultados: Se incluyeron 24 pacientes que ingresaron al Servicio de Urgencias con diagnóstico de TCE severo, realizándose al momento del ingreso una aleatorización mediante tómbola para la integración del grupo experimental $(n=12)$ y el grupo control $(n=$ 12 ), con medias de edad de 26 años para grupo experimental y 49 para grupo control $(p=0.004)$. El promedio de la escala de coma de Glasgow para el grupo experimental al momento del ingreso al estudio fue de 7 puntos y para el grupo control de $6.7(p=0.378)$. La escala de gravedad de APACHE II fue de 19 puntos para el grupo experimental y 13.6 para grupo control $(p=0.052)$ y SOFA al ingreso de 11 puntos grupo experimental y 7 puntos grupo control $(p=$ 0.032 ). Con respecto a los hallazgos tomográficos iniciales, no se observaron diferencias en ambos grupos, en cuanto a desviación de línea media ( $p=0.99)$ y el edema cerebral $(0.99)$; sin embargo, ya integrada la escala de Rotterdam, se observó de 3 y 3.71 puntos para grupo experimental y control, respectivamente $(p=0.007)$. El uso de medidas antiedema cerebral mediante terapia hiperosmolar se empleó en 11 pacientes del grupo experimental y 10 del grupo control $(p=0.99)$. Con relación al punto primario del estudio, se observó una diferencia estadísticamente significativa en los días de estancia en la $\mathrm{UCI}$ con siete días para el grupo experimental y 3.71 días para el grupo control $(p=0.001)$; asimismo, para los días de estancia hospitalaria con 12.5 días y 5.85 días, respectivamente $(p=0.001)$. Para el egreso hospitalario, ambos grupos no mostraron diferencia en la escala de Glasgow Outcome, siendo de 3.50 puntos y 3.57, respectivamente $(0=0.630)$. Finalmente, se observó una mortalidad a los 28 días de un paciente para el grupo experimental y tres pacientes para el grupo control $(p=0.59)$; no obstante, mediante análisis de supervivencia con un modelo de regresión de Cox, se obtuvo un HR de 0.128 (IC 95\% 0.039-0.422), con significancia estadística, además se realizó un análisis por medio de curva de Kaplan-Meier con presencia de mayor supervivencia para el grupo experimental $(p=$ 0.010). Conclusión: El uso de ácido ascórbico en el contexto de TCE severo impactó de manera negativa en la estancia en la $\mathrm{UCI}$, así como en el tiempo de hospitalización. No se encontró diferencia en ambos grupos en cuanto a escalas pronósticas funcionales a su egreso. Sin embargo, el análisis de supervivencia mostró una menor mortalidad a los 28 días para el grupo experimental respecto a los controles, siendo estadísticamente significativo.

Índice de desregulación inmunológica como

predictor de evolución hacia síndrome de

insuficiencia respiratoria progresiva del adulto en

pacientes con neumonía por SARS-CoV-2

Gaytán García Cristhian, Palacios Chavarría Josué Adrián,

Sánchez Aguirre Janet Silvia, Franco Granillo Juvenal

Centro Médico ABC, Ciudad de México, México.

Introducción: Se han descrito los principios fisiopatológicos de la neumonía secundaria a infección por SARS-CoV-2 en donde se identificó la cascada de citocinas como principal factor para el desarrollo de lesiones orgánicas. Actualmente, se realizan mediciones de marcadores bioquímicos inflamatorios en búsqueda de su relación con el pronóstico y posibles complicaciones. En un estudio reciente, se realizó un índice relacionando la interleucina 6 y los niveles de linfocitos y su asociación con la mortalidad en los pacientes con neumonía severa por SARS-CoV-2. El índice de desregulación inmunológica (IL-6/linfocitos) podría permitir estimar a los pacientes que evolucionarán hacia síndrome de insuficiencia respiratoria progresiva del adulto (SIRPA). Objetivos: Establecer si existe una relación entre el nivel de índice de desregulación inmunológica y la aparición de síndrome de insuficiencia respiratoria progresiva del adulto en los pacientes con neumonía por SARS-CoV-2. Resultados: Se analizaron 346 pacientes, el índice de desregulación inmunológica presentó un promedio de 157 en el grupo correspondiente a SIRPA; mientras que en el grupo control el promedio fue de 38. De los pacientes con diagnóstico de SIRPA, 18.6\% fallecieron, mientras que para el grupo control sólo $0.47 \%$. La curva ROC para el análisis de la sensibilidad y especificidad del índice como predictor de evolución hacia SIRPA con un hallazgo de sensibilidad fue de $68.2 \%$ y una especificidad de $77 \%$ con un punto de corte de 99 . Conclusión: La predicción de complicaciones en el contexto de SARS-CoV-2 nos permitirá tomar medidas oportunas; por lo que la existencia de índices predictivos es una herramienta útil, pero que requiere análisis múltiples y validados en distintas poblaciones para contar con un nivel de seguridad alto al tomar decisiones basadas en ellos. En este estudio en particular, el índice de desregulación inmunológica se ha mostrado como un posible predictor de evolución hacia SIRPA; sin embargo, el establecimiento de medidas terapéuticas deberá continuar con relación a hallazgos clínicos, bioquímicos y de imagen.

\section{Certeza diagnóstica del SOFA-simplificado en} pacientes con COVID-19 en Unidad de Terapia Intensiva (UTI) del Centro Médico ABC

Gómez García María Guadalupe, Monares Zepeda Enrique,

Franco Granillo Juvenal, Aguirre Sánchez Janet Silvia, Chaires Gutiérrez Rodrigo, Escala Bejarano María Vigil Centro Médico ABC. Ciudad de México, México.

Introducción: La pandemia de la enfermedad por coronavirus 2019 (COVID-19) continúa desafiando a los sistemas de atención médica en todo el mundo. En los últimos dos meses, sólo unos pocos estu- 
dios han analizado los factores pronósticos de muerte en pacientes con COVID-19. Por ello es de gran relevancia clínica que existan análisis de escalas pronósticas, que ayuden a anteponerse a la gravedad de pacientes, para la toma de decisiones y acciones oportunas que eviten complicaciones y aumento en insumos hospitalarios. En el presente estudio se propone la utilización de escala SOFA-simplificado, la cual es una escala pronóstica que consiste en la evaluación clínica, sin requerimientos de estudios gabinete. Objetivos: Demostrar la certeza diagnóstica mediante la concordancia y grado de acuerdo con utilizar SOFA-simplificado y SOFA. Material y métodos: Estudio de cohorte retrospectiva en pacientes con COVID-19 que ingresaron a la UTI. Se recolectaron datos demográficos, resultados de laboratorio e indicadores inmunológicos, con la finalidad de analizar y comparar escalas pronósticas. Las variables cualitativas se reportan como frecuencias y proporciones. Las variables cuantitativas como mediana con rango intercuartil o media con desviación estándar. Se calcula el tamaño de muestra con Epi Info versión 5.4.6 y OpenEpi. Se compara ambas escalas pronósticas; así como supervivencia de Kaplan-Meier. Se analiza la superposición de ambas escalas para demostrar la certeza diagnóstica mediante curvas de ROC, se valora el área bajo la curva y para la comparación entre ellas se realizará agreement con coeficiente de correlación intraclase $(\mathrm{CCl})$ y representación por BlandAltman. El programa a utilizar para análisis de datos fue IMB SPSS Statistics 24, aplicación Epi Info versión 5.4.6 y OpenEpi. Resultados: El grado de acuerdo medido por índice de correlación intraclase fue de 0.688 ( IC 95\% 0.405-0.836) con p significativa ( $p<0.001$ ), se traduce en un alto grado de acuerdo entre SOFA y SOFA-simplificado y un rendimiento similar. Conclusión: Según el grado de acuerdo tan elevado que se presenta en este estudio, se podría utilizar la escala SOFA-simplificado como sustitución para predecir gravedad en pacientes con COVID en áreas de salud en las que no se cuente con equipo de laboratorio, identificando oportunamente gravedad, mejorar la atención oportuna y disminuir mortalidad.

Hiponatremia y lesión pulmonar: ¿una asociación peligrosa en SARS-CoV-2?

Ledesma Cervantes Lyman, ${ }^{*}$

Sánchez Díaz Jesús Salvador, ${ }^{*}$ Peniche Moguel Karla Gabriela, ${ }^{*}$ Monares Zepeda Enrique, ${ }^{\ddagger}$ González Escudero Eduardo Alberto, ${ }^{*}$

Calyeca Sánchez María Verónica*

${ }^{*}$ Hospital de Especialidades No. 14 CMN «Lic. Adolfo Ruiz

Cortines», IMSS, Veracruz, Veracruz. ${ }^{\ddagger}$ Hospital San Ángel

Inn Universidad, Ciudad de México, México.

Introducción: La pandemia por SARS-CoV-2 (severe acute respiratory syndrome coronavirus 2) se ha convertido en un hito en la historia moderna de la humanidad; la neumonía grave que ocasiona cursa con niveles elevados de IL-6, la cual estimula el hipotálamo para la secreción de hormona antidiurética y condiciona hiponatremia ( $\mathrm{Na}+$ sérico $<135 \mathrm{mEq} / \mathrm{L}$ ), ésta se presenta en $29 \%$ de los pacientes con diagnóstico de neumonía y se considera un marcador de inflamación, gravedad y pronóstico. Material y métodos: Estudio de cohorte, ambispectivo, descriptivo, analítico que incluyó pacientes con diagnóstico de neumonía grave por SARS-CoV-2. Se clasificaron en dos grupos: grupo 1 (G1) sin hiponatremia y grupo 2 (G2) con hiponatremia, y se identificó asociación con la lesión pulmonar. Resultados: Se incluyeron 53 pacientes. El G1 tuvo 37 (69.8\%) y el G2 16 (30.2\%); la media de sodio $\left(\mathrm{Na}^{+}\right)$fue $139.86 \pm 3.55$ y $130 \pm 3.29$ para el $\mathrm{G} 1$ y $\mathrm{G} 2$, respectivamente $(\mathrm{p}=0.001)$. De las variables pulmonares, la $\mathrm{PaO}_{2} / \mathrm{FiO}_{2}$ fue $85.73 \pm 29.93$ y $114 \pm 57.37 \mathrm{mmHg}$, distensibilidad estática de $34.67 \pm 8.99$ y $31.87 \pm 10.89 \mathrm{cmH}_{2} \mathrm{O}$, para el $\mathrm{G} 1$ y G2, respectivamente. La mortalidad en el $\mathrm{G} 1$ fue $42 \%$ y $75 \%$ en el G2, con OR sodio-mortalidad 3.93 (IC 95\% 1.06-14.52), $p=0.03$. Conclusión: Las concentraciones séricas más bajas de $\mathrm{Na}^{+}$están asociadas con mayor severidad y peores resultados en pacientes con neumonía grave por SARS-CoV-2. Convirtiendo a la medición del sodio sérico en un recurso factible de cuantificación para asociarlo con severidad de lesión pulmonar por SARS-CoV-2 e identificar a los pacientes con mayor riesgo de complicaciones y muerte.

Identificación y técnica de medición de las consolidaciones subpleurales mediante ultrasonido en COVID-19

González Martínez Karen Itzel, Montelongo Felipe de Jesús, Islas Ávila Roberto Emmanuel, Gatica Castro David,

González Martínez Diana Vanessa

Hospital General de Ecatepec "Las Américas».

En un principio se pensó que el ultrasonido no era útil para el estudio del pulmón, tomando como base el principio ultrasonográfico en el cual el aire refleja las ondas sonoras actuando como una barrera biológica. Comúnmente, las imágenes de pulmón en pacientes críticamente enfermos se realizan de manera rutinaria por la toma de radiografía de tórax a la cabecera del paciente (RXT) o la de la tomografía computarizada torácica, la cual, aunque es el estándar de oro para la toma de imágenes de pulmón, es cara y no se puede realizar de forma rutinaria. La realización de la radiografía de tórax implica traslado al Departamento de Radiología, lo cual pone en riesgo a un paciente crítico, así como la RXT portátil, que se limita a pobre calidad de imagen y baja sensibilidad, es aquí donde cobra importancia el ultrasonido pulmonar por ser un método fácil, rápido, que puede realizarse a la cabecera del paciente y de menor costo. Objetivo: Proponer un protocolo mediante una técnica ultrasonográfica para identificar las características de las consolidaciones subpleurales y su importancia en la evolución de la enfermedad en pacientes con diagnóstico de COVID-19 en el Departamento de Terapia Intensiva del Hospital General de Ecatepec. Material y métodos: Previa autorización del comité de ética, se realizó un estudio prospectivo analítico en el Departamento de Terapia Intensiva mediante un ultrasonido VINNO 5, con transductor lineal y convexo se identificaron las consolidaciones subpleurales, se tomaron medidas y se siguió la evolución en pacientes ingresados a la Unidad de Terapia Intensiva del Hospital General de Ecatepec. Conclusiones: En este estudio se demostró que las consolidaciones subpleurales son cambios presentes en la infección por SARS-COV 2, y que la identificación y el monitoreo de su tamaño mediante ultrasonido durante el curso clínico de la enfermedad nos pueden dar pauta a la toma de decisiones e inferir en el diagnóstico y pronóstico de dicha patología.

Síndrome de dificultad respiratoria (ARDS) vs síndrome de dificultad respiratoria por COVID-19 (CARDS) las diferencias que realmente importan Bórquez-López Yazmín Fabiola, Monares Zepeda Enrique, Franco Granillo Juvenal, Aguirre Sánchez Janet Silvia, Chaires Gutiérrez Rodrigo

Centro Médico ABC. Ciudad de México, México.

Introducción: El síndrome respiratorio agudo severo causado por el coronavirus 2 (SARS-CoV-2), ahora clasificado como enfermedad por coronavirus 2019 (COVID-19) ha presentado mayor morbilidad y mortalidad en gran parte a la neumonitis viral aguda que evoluciona a síndrome de dificultad respiratoria aguda (SDRA), pero sólo pocos estudios han analizado las comparaciones entre ambas enfermedades. Objetivos: Evaluar las características sociodemográficas, parámetros ventilatorios y mortalidad en pacientes con ARDS vs CARDS. Material y métodos: Estudio de cohorte prospectivo en pacientes con ARDS vs CARDS bajo ventilación mecánica. Resultados: 95 pacientes evaluados, 47 (49.4\%) presentaron ARDS y 48 (50.5\%) presentaron COVID-19. En la comparación de parámetros de oxigenación $\mathrm{PaO}_{2} / \mathrm{FiO}_{2}$ de $134 \mathrm{mmHg}$ (RIC $57-411 \mathrm{mmHg}$ ) en el grupo de ARDS y $118 \mathrm{mmHg}$ (RIC 50-351 $\mathrm{mmHg}$ ) con una $\mathrm{p}=0.746$ en CARDS $\mathrm{sin}$ diferencias significativas, en ambos grupos se respetaron medidas de protección pulmonar con volúmenes tidales bajos $6-8 \mathrm{~mL} / \mathrm{kg}$ peso ARDSNet en ARDS $5.8 \mathrm{~mL} / \mathrm{kg}$ (RIC 4.7-7.8) y CARDS $6.3 \mathrm{~mL} / \mathrm{kg}$ (RIC 4.5-7.5) $p=0.51$, presión meseta $>30 \mathrm{mmHg}$, en CARDS de 24.6 $\mathrm{cmH}_{2} \mathrm{O} \pm 3.2$ y ARDS $24.8 \mathrm{cmH}_{2} \mathrm{O} \pm$ 3.2. $\mathrm{p}=0.810$, presión de conducción $<15 \mathrm{mmHg}$, fue de $13.1 \mathrm{cmH}_{2} \mathrm{O} \pm 3.1$ en ARDS y CARDS de 
$12.9 \pm 2.3 p=0.73$. Con mayor mortalidad $48.9 \%$ y días de ventilación mecánica en pacientes con ARDS. Conclusión: No se encontraron diferencias significativas en ambos grupos respecto al índice de oxigenación, ambos respetaron metas de protección pulmonar, pero se encontró mayor mortalidad y días de ventilación mecánica en el grupo de ARDS.

Infección por SARS-CoV-2 en mujeres con preeclampsia severa en una Unidad de Cuidados Intensivos.

Pronóstico y correlación con la carga viral

Lozano Zúñiga Ramón, Ramos Núñez Manuel,

Hernández Pacheco José Antonio, Gutiérrez Marín Alfredo,

Helguera Repetto Cecilia, León Juárez Moisés, Espino y

Sosa Salvador

Instituto Nacional de Perinatología «Isidro Espinosa de los Reyes».

Objetivo: Comparar el pronóstico de mujeres que ingresaron a la Unidad de Cuidados Intensivos del Instituto Nacional de Perinatología por PE severa e infección por SARS-CoV-2, midiendo la correlación entre la carga viral y las complicaciones maternas. Diseño: Estudio de una cohorte de mujeres con preeclampsia ingresadas en una Unidad de Cuidados Intensivos, por muestreo no probabilístico por caso consecutivo de 105 mujeres en el periodo del 1 de marzo al 15 de octubre del 2020, a quienes se determinó recuento de plaquetas, aminotransferasas, cociente proteínas/creatinina en orina casual $(\mathrm{mg} / \mathrm{dL})$, urea y creatinina, y datos demográficos en las primeras horas de ingreso. Resultados: No existieron diferencias en el número de complicaciones maternas entre las mujeres con PE severa con COVID positivo $(27.5 \%)$ versus las mujeres COVID negativo $(23.6 \%) p=0.58$. Una prueba de COVID positiva no incrementó el riesgo de complicaciones maternas OR $1.31\left(\mathrm{IC}_{95 \%}, 0.495-3.47\right)$. Fue mayor el grado de proteinuria en las mujeres con prueba positiva. Se consideró neumonía asociada con COVID-19 en $27.5 \%$ de las mujeres positivas. Existió una mejor correlación entre las variables de presión arterial y cociente AST/ALT en mujeres con neumonía y la carga viral. Conclusiones: El presente estudio muestra que en mujeres con PE severa atendidas en la Unidad de Cuidados Intensivos no se incrementan las complicaciones maternas cuando existe infección por SARS-CoV-2.

Espacio muerto e índice ventilatorio en el síndrome respiratorio agudo grave por coronavirus tipo 2

Cruz Monzalvo Mónica, Palacios Chavarría Adrián,

Aguirre Sánchez Janet Silvia, Franco Granillo Juvenal

Centro Médico ABC, Ciudad de México, México.

Introducción: El 31 de diciembre de 2019, la Comisión de Salud de la provincia China de Hubei informó por primera vez un grupo de casos inexplicables por neumonía secundaria a un nuevo coronavirus. El virus fue nombrado por la OMS como el nuevo coronavirus SARS-CoV-2, causante de la enfermedad 2019-nCoViD (del inglés, new CoronaVirus Disease). Respecto al síndrome de dificultad respiratoria aguda (SDRA), que provoca falla respiratoria y muerte, es indispensable su diagnóstico y manejo en terapia intensiva. En el manejo del SDRA, la tensión arterial de oxígeno $\left(\mathrm{PaO}_{2}\right)$ entre la fracción inspirada de oxígeno $\left(\mathrm{FiO}_{2}\right)\left(\mathrm{PaO}_{2} / \mathrm{FiO}_{2}\right)$ es la única variable fisiológica medida, el valor pronóstico de $\mathrm{PaO}_{2} / \mathrm{FiO}_{2}$ para predicción de mortalidad es limitado. El aumento de la fracción de espacio muerto es una característica de la fase temprana del SDRA. Los valores elevados están asociados con un mayor riesgo de muerte. De hecho, el aumento de la fracción de espacio muerto (VD/VT) durante la primera semana desde el diagnóstico inicial es un predictor indispensable de supervivencia independiente de la oxigenación. El índice ventilatorio (IV) puede ser calculado de manera rutinaria con variables respiratorias a la cabecera del paciente y se correlaciona positivamente con la fracción de espacio muerto, pudiendo ser un subrogado de ésta. A su vez, medir el IV es reflejo de la eficiencia ventilatoria. Objetivos: Estimar el espacio muerto e índice ventilatorio para valorar respuesta a posición prono en pacientes con SDRA en COVID-19, describir cambios en parámetros ventilatorios en posición prono, valorar la eficiencia ventilatoria. Material y métodos: Población atendida en medicina crítica por SDRA grave. Periodo de reclutamiento: tres meses, cohorte histórica. Se realizó medición de gases arteriales y venosos, pre- y posterior a posición prono. Se realizó cálculo del índice ventilatorio y un análisis univariado de los factores seleccionados, el análisis estadístico se elaboró en XLSTAT y Minitab, se analizaron medidas de frecuencia y se realizó el análisis de los factores de riesgo con prueba de Fisher y $\chi^{2}$. Resultados: La media de edad fue de 56 años, rango de 26 a 51. La distribución por género fue de 25 pacientes del sexo masculino (69.4\%) y 11 del femenino (30.6\%). El promedio de $\mathrm{PaO}_{2}$ fue 98.92, el control, una vez aplicada la maniobra prono, fue de 214.7. En cuanto a los valores de $\mathrm{PaCO}_{2}$, el promedio al inicio de ventilación fue 42.6, control al prono de 43. Se obtuvo medición de $\mathrm{CO}_{2}$ exhalado (ETCO) sólo en nueve pacientes (falta o falla en el equipo de medición) promedio de 42, ETCO prono 37, disminución de porcentaje de cortos (\% VQ) hasta en $50-70 \%$, VQ 36.6, VQ prono 25.31. Índice ventilatorio calculado previo a prono promedio IV 1.68 , IV prono 1.22, se estableció punto de corte de 1.68 como factor asociado con respuesta, $28 \%$ presentó respuesta. Días de ventilación mecánica promedio 11.2, días en la UCl 14.3.

Impacto del decúbito prono en el síndrome de insuficiencia respiratoria aguda en pacientes con COVID-19 bajo ventilación mecánica invasiva González Ruiz Carlos Alejandro, Rentería Díaz Faustino Javier, Martínez Zubieta Ricardo, Cerón Díaz Ulises Wilfrido Hospital Español.

A finales del año 2019 surgió una nueva especie de coronavirus con capacidad de producir enfermedad en humanos, se identificó como COVID-19 y se definió la enfermedad como SARS-CoV-2. A partir de marzo del 2020 comenzaron a presentarse casos de neumonía por SARS-CoV-2 en el Hospital Español de México, algunos de éstos desarrollaron la enfermedad grave y requirieron ventilación mecánica invasiva. Se realizó una serie de casos, de tipo descriptivo, transversal, retrolectivo y analítico, desde mayo hasta agosto de 2020 , en pacientes ingresados en el Área de Terapia Intensiva COVID del Hospital Español de México. Se obtuvo una muestra de 42 pacientes, $32(76 \%)$ hombres y $10(24 \%)$ mujeres, con neumonía severa por SARS-CoV-2 e hipoxemia refractaria con un cuadro de síndrome de insuficiencia respiratoria aguda (SIRA) moderado $\left(\mathrm{PaO}_{2} / \mathrm{FiO}_{2}<\right.$ $200)$ a severo $\left(\mathrm{PaO}_{2} / \mathrm{FiO}_{2}<100\right)$, los cuales necesitaron ventilación mecánica invasiva y por hipoxemia refractaria al menos un evento de decúbito prono. La duración mínima de cada episodio de prono fue al menos de 24 horas, teniendo como mínimo un evento de decúbito y prono y como máximo de siete eventos, con un promedio global de 2.7 eventos de decúbito prono. El tiempo máximo prono por evento fue de 36 horas continuas, teniendo un promedio de 30.1 horas en decúbito prono. Se logró establecer que los pacientes en decúbito prono presentaron una mejoría respecto al índice de oxigenación $\left(\mathrm{PaO}_{2} /\right.$ $\mathrm{FiO}_{2}$ ) con una $\mathrm{p}<0.01$, a diferencia de otros indicadores de oxigenación, tales como $\mathrm{PaO}_{2}$ y $\mathrm{PaCO}_{2}$, no se encontró suficiente evidencia respecto a ser predictor sobre mortalidad y gravedad.

\section{Comparación de la variación del gasto cardiaco por} ultrasonido versus índice de variación pletismográfica en la Unidad de Cuidados Intensivos

Camacho Altamirano Raziel, Montelongo Felipe de Jesús, Tapia Velasco Rafael

Hospital General de Ecatepec Las Américas.

La reanimación en pacientes críticos sigue siendo un tema a debatir. Existen propuestas guiadas en metas que sustentan la individualización de cada paciente con base en sus requerimientos y particularidades especiales. Por eso, existe una amplia gama de propuestas para el beneficio del paciente. Es de vital importancia comentar que una forma idónea de guiar dicha reanimación es mediante la utilización de 
la medición del gasto cardiaco, existiendo métodos invasivos como el Swan-Ganz, métodos semiinvasivos como el PiCCO o métodos no invasivos como la medición de gasto cardiaco mediante ecocardiografía transtorácica. Sin embargo, dentro de las pruebas no invasivas, existen métodos capilares, los cuales han cobrado gran importancia en los pacientes que son sometidos a quirófano, como la utilización de la pletismografía, la cual, en ensayos controlados, ha dado grandes y buenos resultados. El objetivo es comparar la variación del gasto cardiaco contra el índice de variabilidad pletismográfica como medida de respuesta a volumen en los pacientes de la Unidad de Cuidados Intensivos del Hospital General de Ecatepec «Las Américas» del Instituto de Salud del Estado de México. La presente investigación se realizó con el propósito de identificar a aquellos pacientes que sean respondedores a volumen mediante la utilización de la ecocardiografía transtorácica por el método de Bernoulli y compararlo con la medición del índice de variabilidad pletismográfica. Donde encontramos que el ultrasonido tiene una sensibilidad muy elevada para detectar el incremento del gasto cardiaco en aquellos pacientes que sean respondedores a volumen y tienen una especificidad de $52.5 \%$ de detectar a los que sean respondedores a volumen y no requerían del mismo. Por lo tanto, la utiliza- ción de la medición del gasto cardiaco es una herramienta adecuada para guiar la reanimación en pacientes críticos; no obstante, debemos valorar que una adecuada respuesta a volumen no necesariamente se relaciona con la necesidad del mismo. Comparado en el índice de variabilidad pletismográfica, el cual, si bien es muy específico para detectar a aquellos pacientes que son respondedores a volumen, no detecta en los que las condiciones clínicas como el estado de choque disminuyen el llenado capilar, lo que no permite una adecuada interpretación o lectura del dispositivo. Concluimos que la variabilidad del gasto cardiaco mediante ecocardiografía transtorácica, aunque es un método operador dependiente, es muy sensible para determinar si un paciente es respondedor a líquidos o no, aunque no ameriten de dicha administración, comparado con el índice de variabilidad pletismográfica donde no detecta a todos aquellos pacientes que ameritan continuar con reanimación hídrica intensa, sino sólo a aquéllos con un estado de choque o un estado de reanimación más estable, donde el llenado capilar es más efectivo a nivel de los pulpejos; sin embargo, una vez detectado o estabilizado el paciente, sí es efectivo en identificar a aquellos donde se deberá continuar con la reanimación hídrica y a los que ya no se benefician de continuar con dicha reanimación. 